\title{
Symposium review: Oxylipids and the regulation of bovine mammary inflammatory responses ${ }^{1}$
}

\author{
Lorraine M. Sordillo ${ }^{2}$ \\ College of Veterinary Medicine, Michigan State University, East Lansing 48824
}

\begin{abstract}
Inflammation is a critical aspect of the innate immune system that can determine the outcome of several economically important diseases of dairy cattle such as mastitis. The purpose of the inflammatory response is to eliminate the source of tissue injury and then return tissues to normal function. Aggressive or uncontrolled inflammatory responses, however, can damage host tissues and contribute significantly to the pathophysiology associated with mastitis. A precarious balance between pro-inflammatory and pro-resolving mechanisms is needed to ensure optimal pathogen clearance and a prompt return to immune homeostasis. Therefore, inflammatory responses must be tightly regulated to avoid bystander damage to the milk-synthesizing tissues of the mammary gland. Oxylipids are potent lipid mediators that can regulate all aspects of the inflammatory response. The biosynthetic profiles of oxylipids are dependent on both the availability of diverse polyunsaturated fatty acids substrates and their subsequent metabolism through various oxidizing pathways. Changes in lipid metabolism in dairy cows around parturition can profoundly change the composition and concentration of oxylipids in the mammary gland that may be responsible for dysfunctional inflammatory responses during this time. This review will provide a brief overview of the bovine inflammatory response and the role that oxylipids play in contributing to the onset and resolution of inflammation especially as it pertains to mastitis. Factors associated with periparturient cows that can contribute to dysfunctional regulation of inflammation as a function of altered oxylipid biosynthesis and metabolism also will be described. Understanding the role that oxylipids may play in the development of mastitis is key to developing novel prevention and control programs for the dairy industry.
\end{abstract}

\footnotetext{
Received September 18, 2017.

Accepted December 12, 2017.

${ }^{1}$ Presented as part of the ADSA Lactation Symposium: The New and Old of Mammary Gland Physiology and Milk Synthesis at the ADSA Annual Meeting, Pittsburgh, Pennsylvania, June 2017.

${ }^{2}$ Corresponding author: sordillo@msu.edu
}

Key words: immunity, inflammation, mastitis, oxylipin

\section{INTRODUCTION}

The incidence and severity of dairy cattle diseases are greatest during the periparturient period, and health problems occurring during this time can greatly affect the productive efficiency of the entire lactation cycle (Ingvartsen, 2006; Pinedo et al., 2010). Mastitis that occurs around the time of calving is especially problematic due to the deleterious effect that this disease has on milk production, reproductive performance, longevity, and overall dairy herd profitability (Halasa et al., 2007). The ability of dairy cattle to resist the establishment of periparturient diseases such as mastitis is related, in part, to the effectiveness of their inflammatory response (Sordillo and Mavangira, 2014). Inflammation is an essential component of the cow's innate immune system that should function to eliminate infections or other sources of injury, and then restore normal tissue structure and function. For example, an efficient mammary gland inflammatory response should promptly recognize and eliminate mastitis-causing bacteria without causing any noticeable changes to the milk or tissues. A beneficial mammary inflammatory response that is effective against the establishment of new intramammary infections should generally last less than a week once the pathogen is neutralized. Because the inflammatory cascade is meant to be transient, exaggerated or protracted mammary inflammatory responses can result in extensive tissue damage. Indeed, uncontrolled acute and chronic mastitis are excellent examples of dysfunctional inflammatory responses that contribute to mammary immunopathology and significant milk production losses (Halasa et al., 2007; Aitken et al., 2011; Akers and Nickerson, 2011). Thus, optimal mammary gland inflammatory responses should have a rapid onset to neutralize bacteria during the initial stages of tissue invasion but a timely resolution to avoid the immunopathology associated with mastitis.

The onset, magnitude, and resolution of inflammation are controlled by a diverse array of soluble mediators primarily released by resident cells located at the site 
of infection or tissue damage. Some examples of these inflammatory mediators include cytokines, complement, vasoactive amines, and free radicals (Aitken et al., 2011). This review, however, will focus on a specific group of potent lipid-derived inflammatory mediators called "oxylipids," that also are known as oxylipins or eicosanoids in the literature. As the name implies, oxylipids are a family of lipid metabolites derived from the oxygenation of n-6 (omega-6) and n-3 (omega-3) PUFA substrates through both enzymatic and nonenzymatic pathways. Depending on the fatty acid substrate and oxygenation pathway, oxylipids can function to either enhance or resolve the inflammatory cascade (Serhan and Chiang, 2008, Mattmiller et al., 2013). The majority of early studies investigating the role of oxylipids in dairy cattle diseases such as mastitis only investigated arachidonic acid-derived mediators from the cyclooxygenase $(\mathbf{C O X})$ pathway - prostaglandins $(\mathbf{P G})$, leukotrienes $(\mathbf{L T})$, and thromboxanes (TX; Zia et al., 1987; Atroshi et al., 1990). The recent advent of highly sensitive lipidomic technologies, however, has enabled the identification of a complex network of oxylipids that may be produced simultaneously from different PUFA substrates. To date, more than 130 oxylipids that have been identified and many characterized for biological activities (Wang et al., 2014). Thus, the effect that oxylipids may have on coordinating inflammatory responses will depend upon the timing and expression of certain oxylipids profiles during disease processes. This paper will provide a brief overview of the inflammatory response and the role of oxylipids in orchestrating inflammation especially as it pertains to mastitis, and discuss current strategies to alter the biosynthesis of oxylipids with the goal of optimizing inflammation in dairy cows during times of increased susceptibility to mastitis.

\section{ONSET AND RESOLUTION OF INFLAMMATION}

Inflammation is primarily a reaction of the microcirculation that is initiated following exposure of host tissues to microbes and associated microbial components. Resident cell populations located within tissues are able to sense the presence of bacteria through pattern recognition receptors (PRR) that can be located on the cell surface, secreted, or expressed intracellularly. These PRR function by recognizing the diverse array of conserved motifs associated with different groups of microbes that are referred to as pathogen-associated molecular patterns (PAMP; Jungi et al., 2011; Kumar et al., 2011). In the mammary gland, PRR were identified on various leukocyte populations, epithelial cells, and endothelial cells (Strandberg et al., 2005; Porcherie et al., 2012; Ryman et al., 2015b). The toll-like recep- tor (TLR) family of PRR were some of the first to be discovered and are among the best characterized to date. Thirteen different TLR are identified in mammals, of which 10 are known to exist in cattle (Menzies and Ingham, 2006; Kumar et al., 2011). Both TLR2 and TLR4 are abundant PRR during IMI because they are primarily activated in response to PAMP associated with gram-positive (i.e., lipopeptides) and gram-negative (i.e., LPS) mastitis-causing pathogens (Goldammer et al., 2004; Porcherie et al., 2012). The initial PRR-PAMP interaction can trigger multiple pro-inflammatory intracellular signaling pathways including translocation of nuclear factor (NF)- $\kappa \mathrm{B}$ (Liang et al., 2004). During mastitis, NF- $\kappa B$ activation is a major signaling pathway by which soluble mediators are produced to drive the inflammatory cascade (Boulanger et al., 2003; Bannerman, 2009). Cytokines and oxylipids are the principal soluble mediators produced during all stages of the onset and resolution of inflammatory responses. Depending on the type of invading pathogen, the amount and timing of initial soluble mediator production can vary considerably. For example, the expressions of cytokine transcripts were greater and expressed more rapidly in Escherichia coli-infected mammary glands than in Staphylococcus aureus mastitis (Lee et al., 2006). The severity of mastitis also may be associated with specific inflammatory mediator profiles such as excessively high concentrations of tumor necrosis factor- $\alpha$ (TNF- $\alpha$ ) during severe acute coliform mastitis (Sordillo and Peel, 1992). Previous studies also showed that $\mathrm{TXB}_{2}$ and $\mathrm{PGE}_{2}$, known proinflammatory oxylipids, may have important roles in the enhanced severity of $E$. coli mastitis that occurs during the periparturient period (Vangroenweghe et al., 2005). Moreover, increased biosynthesis of $\mathrm{PGE}_{2}$ was related to bacterial growth and systemic disease severity during E. coli mastitis (Pezeshki et al., 2011). Both cytokines and oxylipids have the capacity to interact directly with blood vessels in the mammary gland to alter vascular tone and blood flow within the affected tissues, increase vasodilation of capillaries, and increase vascular permeability needed for the migration of blood leukocytes to the site of injury (Ryman et al., 2015b). In the case of mastitis, neutrophils are the predominant leukocyte type found in milk and mammary tissues during the early stages of infection. The efficiency at which neutrophils are able to phagocytize and kill invading pathogens will influence the establishment of disease (Burvenich et al., 1994; Aitken et al., 2011). The collective responses of the vascular endothelium and infiltration of blood leukocytes into affected tissues can result in some of the classical signs of inflammation that include heat, swelling, redness, pain and loss of function. 
As briefly outlined above, the onset of inflammation to bacterial invasion is a complex and tightly regulated response. Whereas a rapid and robust inflammatory response is protective, however, an uncontrolled acute or chronic inflammatory reaction can lead to extensive tissue damage that is associated with diseases pathogenesis. Indeed, dysfunctional inflammation is now widely appreciated as a common underlying component of many metabolic and infectious diseases that affect periparturient dairy cattle, including mastitis (Sordillo and Mavangira, 2014). Therefore, a timely and natural resolution of inflammation is fundamental to overall dairy cattle health and well-being. The resolution of inflammation is an active event involving specific pro-resolving pathways and mediators that expedite the shut-down process by limiting leukocyte infiltration, modifying soluble mediator production, removing cellular debris, and repairing damaged tissues (Tabas and Glass, 2013; Buckley et al., 2014). An essential requirement to turn off the inflammatory response is the removal of the invading pathogens that were responsible for initiating the inflammatory cascade. The successful neutralization of the inciting pathogen will signal the cessation of proinflammatory mediator synthesis and lead to their catabolism. Because NF- $\kappa \mathrm{B}$ is a major pro-inflammatory pathway activated following detection of bacteria, it makes sense that signaling through this pathway is tightly regulated to prevent sustained activation. An important negative regulator of NF- $\kappa \mathrm{B}$ is the protein complex called inhibitor of $\kappa \mathrm{B}$ (IאB; Liang et al., 2004). In the absence of bacterial stimulation, I $\kappa \mathrm{B}$ is bound to $\mathrm{NF}-\kappa \mathrm{B}$ and retained in the cytoplasm in an inactive form. Activation of the NF$\mathrm{\kappa B}$ signaling pathway is based on the degradation of I $\kappa \mathrm{B}$ that allows for nuclear translocation of NF-kB and leads to pro-inflammatory gene expression. Sustained activation of NF- $\kappa \mathrm{B}$ will normally initiate the expression of I $\mathrm{B}$, which acts as a negative feedback loop. The net effect of enhanced I $\kappa \mathrm{B}$ activity is a decreased expression of proinflammatory factors such as COX-2, IL-1, IL-6, IL-8, and TNF- $\alpha$ (Liang et al., 2004; Gasparini and Feldmann, 2012). Additionally, shifts in NF- $\mathrm{BB}$ and I $\kappa \mathrm{B}$ binding can enhance the expression of antiinflammatory cytokines such as IL-10, transforming growth factor- $\beta$, and IL-1 receptor antagonist, which collectively act to decreases pro-inflammatory mediators and promote tissue repair processes (Gasparini and Feldmann, 2012). In dairy cattle with IMI, studies have shown that TNF- $\alpha$ and IL-1 $\beta$ are expressed rapidly during the initial stages of infection and have potent pro-inflammatory functions, whereas IL-4, IL-10, and IL-17 actively promote the resolution of the inflammatory cascade (Bannerman, 2009).
A relatively new area of research into the termination of inflammation is the production of lipid mediators with potent anti-inflammatory and pro-resolving activities. Studies conducted in human and laboratory species showed that the resolution of inflammation is an active process governed by several distinct families of proresolving oxylipids, which include resolvins, protectins, and lipoxins (LX; Serhan and Chiang, 2008; Bennett and Gilroy, 2016). Although metabolites derived from the COX pathway were traditionally associated with driving the onset of the inflammatory response, several downstream metabolites of COX metabolism including $\mathrm{PGD}_{2}, \mathrm{PGJ}_{2}$, and 15 -deoxy-PGJ ${ }_{2}$ also have the capacity to suppress various pro-inflammatory signaling pathways. Considerable evidence also suggests that certain PG produced during the onset of inflammation, such as $\mathrm{PGE}_{2}$, can serve as negative feedback signals to facilitate the resolution of inflammation (Ricciotti and FitzGerald, 2011). Although these oxylipids will be discussed in detail later in this review, it is important to note here that the active biosynthesis of pro-resolving lipid mediators plays an essential role in limiting neutrophil infiltration into affected tissues, enhancing macrophage clearance of apoptotic cells within affected tissues, and facilitates the restoration of tissues to normal function (Tabas and Glass, 2013). Unfortunately, there is not a great deal of in vivo research available in dairy cattle to suggest how pro-resolving or anti-inflammatory oxylipids may contribute to the resolution of inflammation. The relative expression of plasma oxylipids with known roles in the resolution of inflammation was reported to decrease in transition cows when biomarkers of inflammation are often enhanced (Raphael et al., 2014). Others reported an imbalance between anti- $\left(\mathrm{LXA}_{4}\right)$ and pro- $\left(\mathrm{LTB}_{4}\right)$ inflammatory oxylipids in cows with chronic mastitis due to lower concentrations of $\mathrm{LXA}_{4}$ in the milk (Boutet et al., 2003). A broader understanding of how oxylipids profiles shift to facilitate both the onset and resolution of inflammation is required to design efficacious intervention strategies to optimize bovine inflammatory responses especially during times of increased susceptibility to disease.

\section{OXYLIPID BIOSYNTHESIS}

Typically, oxylipids are not stored within cells, but are rapidly synthesized when needed. Although the biosynthetic pathways involved in the production of different oxylipids can be complex and diverse, the production of oxylipids in general shares some common steps. The initial step in oxylipid biosynthesis is the release of PUFA substrates from membrane phospholipids. The hydrolysis of esterified PUFA is catalyzed 
by members of the phospholipase (PL) enzyme family, especially the calcium-dependent $\mathrm{PLA}_{2}$ (Burke and Dennis, 2009). When cells become activated as a consequence of pathogen exposure or tissue damage, $\mathrm{PLA}_{2}$ is mobilized to release key PUFA precursors that include the n-6 fatty acids [linoleic acid (LA) and arachidonic acid (ArA)] and the $n-3$ fatty acids $[\alpha$-linolenic acid (ALA), eicosapentaenoic acid (EPA), docosapentaenoic acid (DPA), and docosahexaenoic acid (DHA)]. These PUFA are bound to ester linkage in the $s n-2$ position of membrane phospholipids and $\mathrm{PLA}_{2}$ acts as an esterase to facilitate their release (Raphael and Sordillo, 2013).

\section{Enzymatic Oxygenation Pathways}

The oxygenated derivatives of PUFA are produced by 3 major classes of enzymes, including COX, lipoxygenase (LOX), and cytochrome P450 (CYP) epoxygenases. These enzymatic pathways function in general by removing susceptible hydrogen atoms from the PUFA structure with the subsequent insertion of oxygen molecules. The COX pathway is composed of 2 major isoforms (COX-1 and COX-2) that have bifunctional activities. Both isoforms catalyze the initial step in the conversion of ArA to the lipid intermediate $\mathrm{PGH}_{2}$ by extracting a hydrogen atom from the PUFA and transferring it to COX active sites containing tyrosyl residues (Wu et al., 2011). The relatively unstable $\mathrm{PGG}_{2}$ is then reduced at the peroxidase site of COX to form the more stable $\mathrm{PGH}_{2}$, the immediate substrate for a series of cell-specific PG and TX synthases that yield several different biologically active oxylipids (Ricciotti and FitzGerald, 2011). The COX-1 isoform is constitutively expressed in most tissues and synthesizes low levels of $\mathrm{PG}$, such as prostacyclin $\left(\mathrm{PGI}_{2}\right)$, that is primarily involved in the maintenance of normal physiological functions. Conversely, COX-2 is highly inducible in response to NF- $\kappa \mathrm{B}$ activation and is primarily associated with the biosynthesis of pro-inflammatory mediators such as $\mathrm{PGE}_{2}, \mathrm{PGF}_{2 \alpha}$, and $\mathrm{TXA}_{2}$. The relative expression profile of $\mathrm{PG}$ and $\mathrm{TX}$ is important to the character of the inflammatory response because these COX-derived metabolites are known to have opposing actions on vascular tone. For example, $\mathrm{PGI}_{2}$ causes vasodilation and inhibits platelet aggregation, whereas $\mathrm{TXA}_{2}$ promotes aggregation and vasoconstriction. Because both $\mathrm{PGI}_{2}$ and $\mathrm{TXA}_{2}$ are derived from the same intermediate $\mathrm{COX}$ product $\left(\mathrm{PGG}_{2}\right)$, the balanced expression of these oxylipids through different downstream enzymatic pathways is crucial for optimal vascular health and immune capabilities (Ryman et al., 2015a). Indeed, previous studies using bovine endothelial cells showed that oxidative stress can inhibit prostacyclin synthase activity and $\mathrm{PGI}_{2}$ formation, thus disrupting vascular homeostasis (Weaver et al., 2001). Moreover, previous assumptions that all COX-2 metabolites are solely responsible for propagating the inflammatory response are no longer supported by the current literature. For example, ArA-derived $\mathrm{PGE}_{2}$ derived from the COX-2 pathway is recognized as having many proinflammatory activities, including inducing pain and fever. Whereas increased COX-2 expression during the onset of inflammation is typified by $\mathrm{PGE}_{2}$ production, enhanced COX-2 expression during the resolution of inflammation is associated with the presence of the other COX-2-derived ArA metabolites including $\mathrm{PGD}_{2}$ and $15 \mathrm{~d}-\mathrm{PGJ}_{2}$. Both $\mathrm{PGD}_{2}$ and its dehydration end-product $15 \mathrm{~d}_{-} \mathrm{PGJ}_{2}$ can inhibit leukocyte adhesion to endothelial cells and decrease cytokine expression by blocking NF- $\kappa \mathrm{B}$ activation.

There are several different LOX isoforms, including $5-, 8-, 12-$ and 15-LOX, where the nomenclature is defined by the capability of each enzyme to introduce molecular oxygen on a specific carbon of the fatty acid structure (Kuhn and O'Donnell, 2006). The LOX enzymes utilize a nonheme $\mathrm{Fe}^{2+}$ to form ferrous hydroxide that extracts hydrogen and inserts molecular oxygen to form a peroxy radical. The unstable peroxy radical is then rapidly reduced to a peroxy fatty acid. For example, metabolism of ArA by the 5-LOX pathway gives rise to hydroxyl and hydroperoxy derivatives [5-hydroxyeicosatetraenoic acid (HETE) and 5-hydroperoxyeicosatetraenoic acid (HPETE), respectively] that are often elevated during inflammation. The 15LOX isoform is characterized as an inducible enzyme expressed in endothelial cells, epithelial cells, reticulocytes, and macrophages with the ability to oxygenate PUFA during inflammation. The initial oxygenated product formed during AA metabolism by 15-LOX is 15-HPETE, which is the biosynthetic precursor of 15HETE and other LT (Natarajan and Nadler, 2004). Within the larger family of CYP isozymes, a subset of CYP (CYP4A series) uses their heme iron radicals in mediating the epoxygenation and hydroxylation of PUFA to form oxylipids directly or to further metabolize some of the COX-derived metabolites such as $\mathrm{PGE}_{2}$ and $\mathrm{PGD}_{2}$ (Spector et al., 2004).

Although both n-6 and n-3 PUFA can be oxygenated through each of these enzymatic pathways, evidence suggests that some of these pathways may have substrate preferences. For example, n-3 PUFA are relatively poor substrates of COX compared with n-6 PUFA (Wada et al., 2007). In contrast, n-3 PUFA are efficiently metabolized by $5-, 12-$, and 15 -LOX in addition to most CYP450 isoforms. When incubated with equimolar concentrations of different PUFA, CYP enzymes will preferentially oxygenate EPA and DHA 
over LA and ArA (Arnold et al., 2010). There are also considerable differences in substrate preference within PUFA class. For example, it has long been recognized that 15-LOX-1 uses LA as a substrate preferentially over ArA (Soberman et al., 1985). Alternatively, 15LOX-2 has a distinct preference for ArA as a substrate. Although 15-LOX-2 will metabolize LA, it will be at about one-third the efficiency of ArA (Brash et al., 1997). Because mammalian cellular membranes contain different PUFA substrates, the extent of oxygenase activity will depend, in part, on the reaction specificity of COX, LOX, and CYP enzymes.

\section{Nonenzymatic Oxygenation Pathways}

Nonenzymatic pathways of oxygenation are driven by both free radical and nonradical molecules that are often collectively referred to as oxidants. Although the majority of oxidants are reactive oxygen species (ROS), a portion of the total oxidant pool can consist of reactive nitrogen species (Halliwell, 2007). Nonenzymatic oxygenation of PUFA follows the same general mechanisms associated with the major enzymatic pathways of oxylipid biosynthesis. The main difference with the nonenzymatic pathway, however, is that the oxidant-mediated proton removal lacks the stereospecificity demonstrated by each of the enzymatic pathways. Whereas the enzymatic pathways of oxylipid biosynthesis primarily utilize hydrolyzed free PUFA, the main targets of nonenzymatic oxidation pathways are fatty acids that remain esterified to cell membrane phospholipids (Milne et al., 2015). The nonenzymatic oxidation of PUFA is considered a chain reaction consisting of initiation, propagation, and termination, collectively referred to as autoxidation (Yin et al., 2011). The process of autoxidation is initiated during times of oxidative stress that is defined as an imbalance in the production of ROS and the availability of antioxidant defenses needed to neutralize these oxidants (Halliwell, 2007; Sordillo and Aitken, 2009). An important endogenous source of ROS is the by-product of energy production in the mitochondria as part of the electron transport chain, resulting in increased superoxide formation. Periparturient cows often suffer from oxidative stress due to enhanced ROS production during this time of increased metabolic demands associated with the onset of lactation (Bernabucci et al., 2005; Sordillo et al., 2007; Sordillo, 2013). Another major source of ROS is generated by NADPH oxidase activity in phagocytic cells during an inflammatory reaction (Halliwell, 2007). Consequently, cows with mastitis also will experience increased incidence of oxidative stress (Mavangira et al., 2016). Finally, it should be recognized that certain aspects of oxylipid biosynthesis during inflammation also could be a contributing factor to oxidative stress. For example, oxidation of PUFA by COX, LOX, and CYP can produce superoxide in the process that contributes to the total ROS pool (Mavangira and Sordillo, 2018). In addition, initial oxygenation products of enzymatic oxidation are themselves potent hydroperoxides. Metabolism of ArA through the 15-LOX pathway, for example, results in the biosynthesis of 15-hydroperoxyeicosatetraenoic acid (15-HPETE), a potent ROS that has the capacity to cause oxidative damage to cells involved in bovine inflammatory responses (Weaver et al., 2001; Sordillo et al., 2005). Thus, it is not surprising that bovine plasma and milk profiles of oxylipids generated from autoxidation pathways change with respect to lactation stage and during mastitis (Vernunft et al., 2014; Mavangira et al., 2015; Ryman et al., 2015b; Kuhn et al., 2017).

Isoprostanes are a unique series of prostaglandin-like metabolites produced by nonenzymatic peroxidation of PUFA esterified in the $s n-2$ position of membrane phospholipids (Milne et al., 2015). Once formed, isoprostanes are released from phospholipids by $\mathrm{PLA}_{2}$ and often excreted in urine in free form. However, isoprostanes may be found in many animal tissues and plasma in both the free form and esterified to lipoproteins. Two main analytical approaches are used to measure isoprostanes in tissues and biological fluids. The gold standard is gas or liquid chromatography coupled with mass spectrometry due to the high sensitivity and specificity, but there are also many commercially available immunologic-based assays (Lee et al., 2016). The advantage of commercial ELISA is their ease of use and relatively low cost. Unfortunately, ELISA-based analyses are susceptible to cross-reactivity problems due to the structural similarities across the entire prostanoid series of molecules and thus are considered semiquantitative estimations that are often significantly higher than corresponding chromatography and mass spectrometry methods (Klawitter et al., 2011). Thus, the methods used to measure isoprostanes should be carefully considered when comparing studies in the scientific literature and interpreting the information. The classical example of isoprostanes is the family of F2-isoprostanes, especially the 15-F2t-isoprostane (15-F2t-IsoP) isomer that is also sometimes referred to as 8 -isoprostane. The $15-\mathrm{F} 2 \mathrm{t}-\mathrm{IsoP}$ is a product of nonenzymatic peroxidataion of ArA in which ROS generate an ArA peroxyl radical followed by cyclization into an F-pentane peroxyl ring that is then immediately reduced to the stable peroxidation end-product. Sixtyfour different F2-IsoP compounds are derived from ArA that can be detected in biological samples using chromatography and mass spectrometry methods. However, 15-F2t-IsoP was the first and most widely studied of all 
the F2-IsoP compounds and the measure of this isomer is considered a robust biomarker for assessing oxidative stress in vivo (Milne et al., 2015). In human medicine, overproduction of 15-F2t-IsoP is generally regarded as being detrimental to health due to its association with oxidative stress; it enhances plasma expression during several diseases such as cancer, atherosclerosis, and other cardiovascular diseases (Klawitter et al., 2011). Several studies show the direct effects of 15-F2t-IsoP on biological processes associated with dysfunctional inflammatory responses and immunopathology. For example, 15-F2t-IsoP is known to increase platelet aggregation and adhesion in vascular endothelial cells in addition to acting as an $\mathrm{PGF}_{2 \alpha}$ agonist (Pratico et al., 2001). Recent studies in bovine models of oxidative stress and mastitis suggest that $15-\mathrm{F} 2$ t-IsoP is a valid biomarker to assess the degree of lipid peroxidation due to nonenzymatic pathways (Yamada et al., 2013; Vernunft et al., 2014; Mavangira et al., 2016). However, the direct effect that excessive 15-F2t-IsoP may have on bovine cell functions during times of oxidative stress is unknown at this time and additional studies in this area are warranted to fully understand the role of 15-F2t-IsoP in veterinary medicine.

Although IsoP generated from ArA are often associated with oxidative stress and disease, there is now an appreciation that certain n-3-derived IsoP have some beneficial health effects in humans. Although all n-3 PUFA are susceptible to peroxidation, recent evidence suggests that IsoP derived from ALA (F1-phytoprostanes, F1-PhytoP), EPA (F3-IsoP), and DHA (Fneuroprostanes) have beneficial biological functions as well as being excellent biomarkers of oxidative stress in humans (Joumard-Cubizolles et al., 2017). Although $\mathrm{n}$-3-derived IsoP are in relatively low concentrations compared with the n-6-derived 15-F2t-IsoP, they can be readily detected in vivo during times of oxidative stress in human and murine models of disease. The linkage between the enhanced expression of n-3-derived IsoP and disease, however, is not necessarily a disadvantage because several in vitro and in vivo studies demonstrate a direct health benefit of $n-3$ peroxidation. For example, several isoforms for the phytoprostanes can inhibit NF- $\kappa \mathrm{B}$ activation and translocation, reduce the expression of IL-12, and decrease platelet aggregation in human vascular endothelial cells (Pratico et al., 2001). Similarly, F3-IsoP and F-neuroprostanes were shown to inhibit NF- $\mathrm{BB}$ activation, reduce IL-1 and TNF- $\alpha$ production, and decrease leukocyte adhesion with in vitro models using in human endothelial cells and monocytes (Joumard-Cubizolles et al., 2017). Collectively, the n-3-derived IsoP are considered as not only sensors of oxidative stress, but also early lipid mediators that contribute to the proper cellular response to tissue damage. In addition to the isoprostanes, it should be noted that nonenzymatic oxidation of LA and ALA can also produce hydroperoxide metabolites such as HPETE and hydroperoxoctadecaienoic acid (HPODE), respectively. Different from the enzymatic formation of ALA and LA derived oxylipids, certain metabolites such as 11-HETE and 9-hydroxyoctadecadienoic acid (9-HODE) are produced nonenzymatically and can be used as proxies during times of oxidative stress. Although nonenzymatic mechanisms of oxylipid formation are often overlooked as a source of biological active lipid mediators, it is important to note that both n- 6 and n-3 fatty acids give rise to dozens of oxylipid metabolites, including hydroxyperoxides and isoprostanes that control a broad range of biological functions, many of which have yet to be defined in dairy cattle.

\section{Oxylipid Metabolism}

Another important control point in the biosynthesis of oxylipids is the degree to which the initial oxygenation product is subsequently metabolized. The initial product resulting from either enzymatic or nonenzymatic fatty acid oxidation is subjected to additional downstream metabolism to form a diverse network of oxylipids. As an example, the intermediate product from the metabolism of AA through the either the COX-1 or COX-2 pathway is $\mathrm{PGH}_{2}$. This intermediate metabolite serves as the common substrate for a series of specific isomerase and synthase enzymes that ultimately produce $\mathrm{PGE}_{2}, \mathrm{PGI}_{2}, \mathrm{PGD}_{2}$, $\mathrm{PGF}_{2 \alpha}, \mathrm{TXA}_{2}$, and $\mathrm{TXB}_{2}$. Because all prostanoids are derived from a single precursor metabolite $\left(\mathrm{PGH}_{2}\right)$, the relative expression of $\mathrm{PG}$ and TX during health and disease will largely be determined by the differential expression and activity of their respective biosynthetic enzymes. For example, both PGI and PGE synthases are often co-induced along with COX-2 at sites of inflammation. Indeed, recent studies showed that increased $\mathrm{PGE}_{2}$ and $\mathrm{PGI}_{2}$ biosynthesis corresponded to enhanced COX-2 transcript expression in Streptococcus uberis-infected bovine mammary tissues (Ryman et al., 2015b). Evidence suggests that $\mathrm{PGE}_{2}$ may also play an important role in triggering the resolution of inflammation by enhancing LOX activity and the subsequent formation of the ArA-derived lipoxin $\mathrm{A}_{4}\left(\mathrm{LXA}_{4}\right)$ that has potent anti-inflammatory properties (Serhan and Chiang, 2008). Lipoxins are generated by a process of transcellular biosynthesis involving the sequential actions of LOX from at least 2 different cell types. For example, the initial oxygenation of ArA through the 15-LOX pathway in human epithelial cells generates a 15-HETE precursor that is then metabolized through the 5-LOX pathway in macrophages to produce $\mathrm{LXA}_{4}$ 
and $\mathrm{LXB}_{4}$. Conversely, ArA metabolism by 5 -LOX in leukocytes and the release of $\mathrm{LTA}_{4}$ can be converted by 15-LOX in platelets for LX biosynthesis (Serhan et al., 2008). Changes in the timing and ratio of oxylipid biosynthesis through theses LOX pathway were shown to be significant in dairy cattle health. Relative to mastitis, for example, an imbalance of $\mathrm{LXA}_{4}: \mathrm{LTB}_{4}$ occurs during chronic mastitis and was reportedly due to the dramatic reduction in $\mathrm{LXA}_{4}$ biosynthesis within infected mammary glands (Boutet et al., 2003).

Changes in the redox status of cells also can influence the degree to which initial oxygenation products of PUFA are metabolized to form downstream lipid mediators. Oxidation of LA by $15-\mathrm{LOX}-1$ results in the initial product 13-hydroperoxoctadecaienoic acid (13-HPODE), which can be reduced to 13-hydroxyoctadecadienoic acid (13-HODE) by antioxidants and reducing agents such as glutathione (Kuhn et al., 2015). Then, 13-HODE can be metabolized further to 13-oxooctadecadienoic acid (13-oxoODE) by the actions of NADPH-dependent fatty acid dehydrogenase (Altmann et al., 2007). The significance of the sequential oxidation and metabolism of LA is that the pro-inflammatory 13-HPODE is required for the subsequent generation of the anti-inflammatory 13-oxoODE. Current literature supports the contention that 13-HPODE biosynthesis is closely aligned with aberrant inflammatory responses and the pathology of several inflammatory-based disease (Kuhn et al., 2015). Because HPODE are unstable and rapidly reduced during sample processing, they are combined into a single HODE fraction during quantification that makes deciphering biological functions a challenge. Indeed, a recent study showed that 13-HODE was the most abundant plasma oxylipid in early lactation dairy cows and this oxylipid was correlated with gene expression of IL-12 and inducible nitric oxide synthase in peripheral blood leukocytes (Raphael et al., 2014). In the same study, the conversion of 13-HODE to 13-oxoODE was also decreased in early-lactation cows compared with cows in later stages of lactation (Raphael et al., 2014). The significance of diminished metabolism of 13-HODE to 13-oxoODE on inflammatory responses around the time of calving is not known at this point, but it is possible that excessive 13-HPODE or 13-HODE accumulation during the periparturient period may contribute to dysfunctional inflammatory responses. In support of this conjecture, the biosynthesis of 13-HPODE/13HODE and 13-oxoODE was increased significantly in milk and mammary tissues obtained from cows with either Streptococcus uberis or Escherichia coli mastitis (Mavangira et al., 2015; Ryman et al., 2015b). The extent to which 13-HPODE metabolism could contribute to the pathogenesis of mastitis was examined further in a series of in vitro studies using bovine mammary endothelial models. Exposure of cultured mammary endothelial cells to physiological doses of 13-HPODE significantly increased the expression of pro-inflammatory gene expression, but exposure to 13-HODE had no effect (Ryman et al., 2015b). Moreover, exposure to 13-HPODE but not 13-HODE significantly reduced endothelial barrier integrity by increasing bovine mammary endothelial cell apopotosis (Ryman et al., 2016). Changing the redox environment by exposing endothelial cells to an antioxidant during 13-HPODE treatment prevented the adverse effects on endothelial barrier integrity, which emphasizes the need to reduce 13-HPODE to the less-reactive 13-HODE during inflammation (Ryman et al., 2016).

The CYP epoxygenase pathways is another example of how oxylipid profiles reflect the degree of downstream metabolism following biosynthesis of the initial oxidation product. Isoforms of the CYP epoxygenase pathway (CYP2J and CYP2C) metabolize ArA to form 4 epoxyeicosatrienoic acid (EET) regioisomers that are thought to play a significant role in regulating inflammatory responses. For example, 11,12-EET was shown to significantly attenuate endothelial cell activation and leukocyte-endothelial interactions in several human and mouse cell culture systems (Deng et al., 2010). All 4 of the EET regioisomers (5,6-, 8,9-, 11,12-, and 14,15 -EET) were able to attenuate IkB- $\alpha$ degradation following TNF- $\alpha$ stimulation of bovine aortic endothelial cells (Liu et al., 2005). Soluble exopide hydrolase (sEH) can rapidly hydrolyze EET to their corresponding dihydroxyeicosatrienoic acid (DHET) metabolites that generally have much less biological activity. The significance of the further metabolism of EET by sEH is that many of the beneficial anti-inflammatory functions of the initial epoxygenase metabolites are lost. Indeed, several in vivo and in vitro murine models utilize sEH inhibitors to potentiate the antiinflammatory properties of specific EET (Deng et al., 2010; Gabbs et al., 2015). To date, there is no information of how manipulation of sEH activity in dairy cows may influence the pathogenesis of inflammatory-based diseases such as mastitis. Given the predominant shift in CYP-derived metabolites during coliform mastitis (Mavangira et al., 2015), future studies are warranted to examine in more detail how subsequent metabolism of initial CYP-derived epoxygenases might influence disease pathogenesis.

\section{Cell and Tissue Specificity of Oxylipid Biosynthesis}

Another important aspect influencing the expression of oxylipid is that many of the requisite enzymes, PUFA substrates, and downstream metabolites will be 




Figure 1. Oxylipid biosynthesis: PUFA, including linoleic acid, linolenic acid, and their respective derivatives, serve as the substrates for oxylipid biosynthesis. Esterified PUFA are released from the membrane phospholipid through the actions of phospholipase A2. Once released from the membrane, PUFA are oxidized through enzymatic pathways (cyclooxygenase, lipoxygenase, cytochrome P450 epoxygenases, and cytochrome P450 w-hydroxylases) or nonenzymatic pathways (reactive oxygen species and free radicals). The initial oxygenation products may then be subjected to additional metabolism by isomerases, dehydrogenases, or hydrolases. Various downstream metabolites can have either proinflammatory or pro-resolving effects on inflammatory responses. Color version available online.

specific to particular types of cells located in various tissues through the body. For example, stimulation of bovine monocytes with heat-killed Strep. uberis or LPS resulted in a more robust increase in 15-LOX1 gene expression and subsequent 13-HPODE and 13-HODE biosynthesis compared with mammary endothelial cells (Ryman et al., 2016). Therefore, the production of specific oxylipids will vary depending on cell type. Previous studies also showed that bovine milk oxylipid profiles were different from plasma profiles detected during different stages of lactation (Kuhn et al., 2017). Similarly, plasma profiles of oxylipids were different from those observed in mammary tissues or milk during clinical bovine mastitis (Mavangira et al., 2015; Ryman et al., 2015b). As such, systemic changes in oxylipids during health and disease are not necessarily reflective of profiles at the tissue level. A better understanding of the cellular sources of oxylipids and the effect that the microenvironment may have on lipid mediator profiles could lead to more targeted therapies for inflammatorybased diseases such as mastitis.

\section{TARGETING OXYLIPID BIOSYNTHESIS}

Although inflammatory responses can protect dairy cattle against infections and injury, we now recognize that dysfunctional inflammatory responses play a major role in the pathophysiology of several health disorders, including mastitis (Sordillo and Mavangira,
2014). Oxylipids represent a complex network of biochemical pathways that are activated during tissue insult to control the onset, magnitude, and duration of the inflammatory response. As such, considerable research effort has been focused on designing strategies to control aberrant inflammatory responses through the regulation of oxylipid biosynthesis. The 2 generalized approaches to manipulating oxylipids biosynthesis have been either to target mechanisms that promote inflammation or to enhance endogenous pathways that promote the resolution process. Whether targeting the onset or resolution of inflammation, intervention strategies to modify oxylipid biosynthesis have addressed several layers of regulation, including substrate availability, modification of enzymatic and nonenzymatic pathways of oxidation, and influencing the degree of oxylipid metabolism (Figure 1).

\section{Modification of PUFA Substrates}

Alterations in cellular fatty acid composition can modify immune responses in several ways, as extensively studied in human and rodent models (Calder, 2002). Once internalized by leukocytes and endothelial cells, certain fatty acids can play a significant role in intracellular signaling pathways that can regulate the magnitude and duration of inflammation. In humans, for example, palmitate and stearate can enhance proinflammatory signaling pathways through the activa- 
tion of NF- $\mathrm{BB}$ (de Heredia et al., 2012). In contrast, a variety of n-3 PUFA, including linolenic acid, EPA, and DHA, are natural ligands for peroxisome proliferatoractivated receptors that can downregulate inflammatory reaction in many cell types including monocyte/ macrophages and endothelial cells (Calder, 2002; de Heredia et al., 2012). Although the direct effects that plasma fatty acids may have on these signaling pathways was not examined specifically in the dairy cow, it is interesting to note that changes in the composition of bovine plasma fatty acids around calving are consistent with the fatty acid composition of human plasma that favors a pro-inflammatory response (Contreras et al., 2012b; de Heredia et al., 2012).

Another way that cellular fatty acid content can influence the inflammatory response is through the biosynthesis of lipid mediators. Oxylipids are derived from PUFA in cellular membranes, and it has been recognized for some time that the relative abundance or ratio of n- 6 and n-3 fatty acids can influence lipid mediator biosynthesis that control inflammation and disease pathogenesis (Calder, 2002; Serhan and Chiang, 2008). Based on promising findings in human medicine, there is an interest in harnessing the health benefits of n-3 PUFA to control inflammatory-based diseases in dairy cattle. Unlike in humans, however, the ability to extrapolate the amount of PUFA consumed with amounts and n-6:n-3 ratio actually incorporated into tissues is not straightforward due to the loss of PUFA through biohydrogenation in the rumen (Raphael and Sordillo, 2013). Yet, several studies have investigated the possibility of supplementing diets of dairy cattle with commercially available rumen-protected PUFA in the form of calcium salts with the goal of altering production efficiency and improving milk yields. For example, increasing dietary intake of calcium salts of fish oil, which is rich in both EPA and DHA, improved reproductive performance by increasing fertility and mitigating pregnancy losses by reducing uterine concentrations of $\mathrm{PGF}_{2 \alpha}$ (Mattos et al., 2004; Dirandeh et al., 2013). The direct effect of altering the n-6 and n-3 PUFA content of immune cells was also assessed in a series of experiments using cultured bovine endothelial cells. The in vitro exposure of bovine endothelial cells to nonesterified fatty acid mixtures that mimic the composition and concentration found in cows during the periparturient period not only increased the production of LA-derived 9- and 13-HODE, but also caused the upregulation of pro-inflammatory cytokines and adhesion molecule transcript expression (Contreras et al., 2012b). The pro-inflammatory response of endothelial cells could be reduced by increasing the n- 3 content of the nonesterified fatty acid mixtures possibly due to shifts in oxylipid profiles as there were significant changes in the expression of pro-resolving oxylipids such as resolvins, protectins, and lipoxins (Contreras et al., 2012a). More recently, cows supplemented with n-3 PUFA through direct infusion into the abomasum could effectively modify both plasma and blood leukocyte PUFA content. When these blood leukocytes were challenge ex vivo with heat-killed Strep. uberis or LPS, the potent anti-inflammatory 15-oxoODE and $\mathrm{LXA}_{4}$ were induced (Ryman et al., 2017). Collectively, the current literature supports the concept that supplementing the diets of cows with PUFA can affect oxylipid biosynthesis and effectively alter the functional capacity of cells involved in immune and inflammatory responses.

\section{Modification of Enzyme Activities}

The initial control point in the biosynthesis of most oxylipids is the release of PUFA substrate from the membrane phospholipid by the actions of $\mathrm{PLA}_{2}$. Increased concentrations of $\mathrm{PLA}_{2}$ can be detected in plasma or other biological fluids of patients suffering from various inflammatory-based diseases (Gabbs et al., 2015). Glucorticoids are widely used to treat dysfunctional acute and chronic inflammatory responses. Although the anti-inflammatory effects are mediated through several pathways, the ability to alter oxylipid biosynthesis by regulating $\mathrm{PLA}_{2}$ activity is a major mode of action (Triggiani et al., 2009). In fact, glucocorticoids have the capacity to inhibit the synthesis of a broad range of PG, TX, and LT oxylipids with major pro-inflammatory functions. The uses of synthetic inhibitors of $\mathrm{PLA}_{2}$ have been studied extensively in human medicine to control the biosynthesis of proinflammatory oxylipids and oxidative stress. They function through different mechanisms of action, including altering the enzymatic activities of $\mathrm{PLA}_{2}$ by changing the physiochemical properties of phospholipid biolayers or by acting on allosteric sites on the enzyme to modify activity. Unfortunately, even the most selective $\mathrm{PLA}_{2}$ inhibitors were reported to product undesirable nonspecific effects that preclude their utility, as is typical when drug discovery is faced with a complex network of enzymatic activity (Dennis and Norris, 2015).

The second important control point in the biosynthesis of oxylipids involves the enzymes responsible for converting PUFA to the initial molecule in the relevant pathway. The nonsteroidal anti-inflammatory drugs (NSAID) are some of the most widely used pharmacological tools that function by blocking oxylipid synthesis through inhibiting the COX pathways. Most NSAID compounds, such as aspirin, ibuprofen, and naproxen, are inhibitors of both COX-1 and COX isoforms. Each of these NSAID have unique modes of action. For example, aspirin covalently alters the enzymes, 
which abolishes COX activity while leaving peroxidase activity intact. Alternatively, ibuprofen and naproxen function as reversible inhibitors of COX activity. The success in treating various human inflammatory conditions with NSAID has validated the targeting of COX activity. However, gastrointestinal toxicities are associated with widespread NSAID use due to inhibition of gastroprotective PG produced via the COX-1 pathway and this has proven to be a major limitation during long-term therapy. The subsequent development of selective COX-2 inhibitors, such as celecoxib and rofecoxib, aimed to retain the anti-inflammatory and analgesic effects of the traditional NSAID without the adverse gastrointestinal issues. Prolonged use of selective COX-2 inhibitors, however, was found to have adverse cardiovascular events, likely due to an imbalance between prothrombotic $\mathrm{TXA}_{2}$ and antithrombotic $\mathrm{PGI}_{2}$ (Rao and Knaus, 2008). In veterinary medicine, NSAID are used in dairy cows to manage the inflammation and alleviate the pain associated with mastitis. Compared with control animals receiving no treatment, the use of flunixin meglumine was shown to decrease rectal temperatures, decrease signs of inflammation, and maintain rumen motility in cows with LPS-induced clinical mastitis. When treating natural acute toxic mastitis cases, both ketoprofen and flunixin meglumine have been used as supportive therapy and both were found to be effective in alleviating pain and improving health variables (Leslie and Petersson-Wolfe, 2012). The efficacy associated with the use of NSAID therapy for mastitis is not always consistent, however, and it is likely that the timing of administration relative to the onset of inflammation plays a major role in the success or failure of the treatment. Future studies that examine oxylipid profiles during an effective inflammatory response and how the balanced expression of proand anti-inflammatory oxylipids change during disease pathogenesis may provide new insights into maximizing the effectiveness of NSAID treatment.

The 5-LOX pathway is a major drug target for the control of aberrant inflammatory responses and the inhibitor zileuton has the ability to block the biosynthesis of all downstream metabolite formation (Dennis and Norris, 2015). Leukotrienes act in a paracrine fashion through interaction with G-protein coupled receptors (GPCR), of which 4 have been cloned to date, consisting of the cysteinyl leukotriene receptors (CysLT1 and CysLT2) and the LTB4 receptors (BLT1 and BLT2). Other strategies to control the actions of the 5-LOX pathway is by the use of LT receptor antagonists that can effectively inhibit CysLT1 (Dennis and Norris, 2015). However, treatment strategies that target only a single enzymatic oxygenation pathway have some significant limitations due to unexpected effects on the entire oxylipid network. For example, blocking the COX pathway with NSAID will result in the switching of PUFA oxidation to other pathways such as 5-LOX. This observation has given rise to the concept of a polypharmacological approach, in which drugs target several different stages in the biosynthesis of oxylipids (Meirer et al., 2014). An example of capitalizing on the crosstalk within the oxylipid network would be combining NSAID that result in the increase in LT metabolites with 5-LOX inhibitors or the use of LT receptor antagonist that may be more effective (Dennis and Norris, 2015). Similar strategies were used in the treatment of bovine mastitis where a combination of COX inhibitors (flunixin meglumine) and LOX inhibitor (nordihydroguaiaretic acid) was efficacious in decreasing $\mathrm{PGE}_{2}$ and $\mathrm{TXA}_{2}$ concentraion in milk and improved the clinical symptoms of inflammation (Rose et al., 1991).

\section{Modifying Degree of Metabolism}

Oxidative stress and oxylipid biosynthesis are tightly linked. Changes in redox status and the development of oxidative stress are known to have profound effects on oxylipid profiles, especially during inflammatory events (Mattmiller et al., 2013). Likewise, oxylipid biosynthesis has the capacity to contribute to oxidative stress by contributing to local ROS pools. This is accomplished through several mechanisms that include superoxide by product formation during the enzymatic oxidation of PUFA and by the formation of potent lipid hydroperoxides as the initial oxygenation product of lipid metabolism (Mavangira and Sordillo, 2018). As such, there is potential for oxylipid biosynthesis and oxidative stress to form deleterious feedback loops that can exacerbate inflammation and enhance the immunopathology associates with diseases such as mastitis (Aitken et al., 2011). There is a growing interest in trying to control both oxidative stress and oxylipid biosynthesis through nutritional intervention (Sordillo, 2016). An area that holds considerable promise is the modification of oxylipid biosynthesis by antioxidant micronutrient supplementation. In both human and veterinary medicine to date, most of this research has focused on dietary selenium (Se) and its effect on the biosynthesis of a diverse array of oxylipids in several different species (Mattmiller et al., 2013). A study conducted decades ago showed that dairy cows deficient in Se had significantly more pro-inflammatory $\mathrm{TXB}_{2}, \mathrm{PGE}_{2}$, and $\mathrm{LTB}_{4}$ in mastitis milk compared with cow on a Se-sufficient diet (Maddox et al., 1991). Subsequent studies using bovine endothelial cells showed that reduced Se status not only caused oxidative stress, but also increased the expression of 15-HPETE, a highly reactive hydro- 
peroxy intermediate formed during the metabolism of ArA through the 15-LOX1 pathway (Cao et al., 2000; Weaver et al., 2001; Trigona et al., 2006). Moreover, the increased accumulation of 15-HPETE during Se deficiency increased the pro-inflammatory phenotype and rates of apoptosis in bovine endothelial cells and murine macrophages (Sordillo et al., 2005; Mattmiller et al., 2014). The influence that Se nutritional status has on oxylipid biosynthesis is due to the relative expression the selenoproteins and their effect on regulating the intracellular redox environment (Mattmiller et al., 2013). Redox regulation by glutathione peroxidase and thioredoxin reductase couples was shown to have control over several levels of oxylipid biosynthesis, including the expression of oxidizing enzymes (COX, LOX, and CYP), the activity of select PG and TX synthases, and the capacity needed to reduce highly reactive lipid hydroperoxides to their less-reactive hydroxyl product (Mattmiller et al., 2013). A better understanding of how micronutrients with the capacity to regulate intracellular oxidant status can regulate oxylipid profiles will lead to more efficient and consistent nutritional strategies to optimize host inflammatory responses.

\section{CONCLUSIONS}

During uncontrolled inflammation, a likely combination of enhanced production of pro-inflammatory oxylipids and reduced expression of anti-inflammatory oxylipids prevents the full resolution of inflammation and the return to immune homeostasis. With recent improvements in lipidomic methodologies, it is now possible to explore comprehensively how oxylipid networks change during health and disease. Historically, it was thought that oxylipids derived from n-6 PUFA (i.e., PG, LT, and TX) were primarily pro-inflammatory, whereas oxylipids biosynthesized from n-3 PUFA (i.e., protectin and resolvins) primarily promoted the resolution of inflammation. With advances in analytical capabilities, it is now widely recognized that these earlier assumptions were too simplistic and that the oxylipid network is complex, highly interactive, and often cell-specific in orchestrating the onset or resolution of inflammatory responses. At present, ample evidence in dairy cattle suggests that oxylipid biosynthesis is controlled at several levels, including the amount and availability of PUFA substrates, the relative activity and substrate preference of oxidizing enzymes, and the degree to which intermediate metabolites are catabolized to their end products. The biosynthetic profiles of oxylipids and the subsequent effect that these metabolites have on the character of the inflammatory response also likely depend on the timing of their subsequent metabolism through various oxidizing pathways. The simultaneous evaluation of diverse pathways of oxylipid biosynthesis has already provided new opportunities to evaluate dysfunctional inflammatory responses that may be unique to specific cells or affected tissues. A greater understanding of the factors that can regulate the delicate balance between the initiation and resolution of inflammatory responses is needed to diminish the morbidity and mortality associated with health disorders of dairy cattle such as mastitis.

\section{ACKNOWLEDGMENTS}

This work was funded in part by grants (2014-6800421972 and 2017-67015-26676) from the Agriculture and Food Research Initiative Competitive Grants Programs of the USDA National Institute for Food and Agriculture (Washington, DC) and by an endowment from the Matilda R. Wilson Fund (Detroit, MI).

\section{REFERENCES}

Aitken, S. L., C. M. Corl, and L. M. Sordillo. 2011. Immunopathology of mastitis: Insights into disease recognition and resolution. J. Mammary Gland Biol. Neoplasia 16:291-304.

Akers, R. M., and S. C. Nickerson. 2011. Mastitis and its impact on structure and function in the ruminant mammary gland. J. Mammary Gland Biol. Neoplasia 16:275-289.

Altmann, R., M. Hausmann, T. Spöttl, M. Gruber, A. W. Bull, K. Menzel, D. Vogl, H. Herfarth, J. Schölmerich, W. Falk, and G. Rogler. 2007. 13-Oxo-ODE is an endogenous ligand for PPAR $\gamma$ in human colonic epithelial cells. Biochem. Pharmacol. 74:612-622.

Arnold, C., A. Konkel, R. Fischer, and W. H. Schunck. 2010. Cytochrome P450-dependent metabolism of omega- 6 and omega-3 longchain polyunsaturated fatty acids. Pharmacol. Rep. 62:536-547.

Atroshi, F., S. Sankari, A. Rizzo, T. Westermarck, and J. Parantainen. 1990. Prostaglandins, glutathione metabolism, and lipid peroxidation in relation to inflammation in bovine mastitis. Adv. Exp. Med. Biol. 264:203-207.

Bannerman, D. D. 2009. Pathogen-dependent induction of cytokines and other soluble inflammatory mediators during intramammary infection of dairy cows. J. Anim. Sci. 87(Suppl):10-25.

Bennett, M., and D. W. Gilroy. 2016. Lipid mediators in inflammation. Microbiol. Spectr. 4:MCHD-0035-2016.

Bernabucci, U., B. Ronchi, N. Lacetera, and A. Nardone. 2005. Influence of body condition score on relationships between metabolic status and oxidative stress in periparturient dairy cows. J. Dairy Sci. 88:2017-2026.

Boulanger, D., F. Bureau, D. Melotte, J. Mainil, and P. Lekeux. 2003. Increased nuclear factor kappaB activity in milk cells of mastitisaffected cows. J. Dairy Sci. 86:1259-1267.

Boutet, P., F. Bureau, G. Degand, and P. Lekeux. 2003. Imbalance between lipoxin A4 and leukotriene B4 in chronic mastitis-affected cows. J. Dairy Sci. 86:3430-3439.

Brash, A. R., W. E. Boeglin, and M. S. Chang. 1997. Discovery of a second 15S-lipoxygenase in humans. Proc. Natl. Acad. Sci. USA 94:6148-6152.

Buckley, C. D., D. W. Gilroy, and C. N. Serhan. 2014. Proresolving lipid mediators and mechanisms in the resolution of acute inflammation. Immunity 40:315-327.

Burke, J. E., and E. A. Dennis. 2009. Phospholipase $\mathrm{A}_{2}$ biochemistry. Cardiovasc. Drugs Ther. 23:49-59.

Burvenich, C., M. J. Paape, A. W. Hill, A. J. Guidry, R. H. Miller, R. Heyneman, W. D. Kremer, and A. Brand. 1994. Role of the neutrophil leucocyte in the local and systemic reactions during 
experimentally induced $E$. coli mastitis in cows immediately after calving. Vet. Q. 16:45-50.

Calder, P. C. 2002. Dietary modification of inflammation with lipids. Proc. Nutr. Soc. 61:345-358.

Cao, Y. Z., C. C. Reddy, and L. M. Sordillo. 2000. Altered eicosanoid biosynthesis in selenium-deficient endothelial cells. Free Radic. Biol. Med. 28:381-389.

Contreras, G. A., S. A. Mattmiller, W. Raphael, J. C. Gandy, and L. M. Sordillo. 2012a. Enhanced n-3 phospholipid content reduces inflammatory responses in bovine endothelial cells. J. Dairy Sci. 95:7137-7150.

Contreras, G. A., W. Raphael, S. A. Mattmiller, J. Gandy, and L. M. Sordillo. 2012b. Nonesterified fatty acids modify inflammatory response and eicosanoid biosynthesis in bovine endothelial cells. J. Dairy Sci. 95:5011-5023.

de Heredia, F. P., S. Gomez-Martinez, and A. Marcos. 2012. Obesity, inflammation and the immune system. Proc. Nutr. Soc. 71:332338.

Deng, Y., K. N. Theken, and C. R. Lee. 2010. Cytochrome P450 epoxygenases, soluble epoxide hydrolase and the regulation of cardiovascular inflammation. J. Mol. Cell. Cardiol. 48:331-341.

Dennis, E. A., and P. C. Norris. 2015. Eicosanoid storm in infection and inflammation. Nat. Rev. Immunol. 15:511-523.

Dirandeh, E., A. Towhidi, Z. Ansari Pirsaraei, F. Adib Hashemi, M Ganjkhanlou, S. Zeinoaldini, A. Rezaei Roodbari, T. Saberifar, and H. V. Petit. 2013. Plasma concentrations of PGFM and uterine and ovarian responses in early lactation dairy cows fed omega-3 and omega-6 fatty acids. Theriogenology 80:131-137.

Gabbs, M., S. Leng, J. G. Devassy, M. Monirujjaman, and H. M. Aukema. 2015. Advances in our understanding of oxylipins derived from dietary PUFAs. Adv. Nutr. 6:513-540.

Gasparini, C., and M. Feldmann. 2012. NF-kappaB as a target for modulating inflammatory responses. Curr. Pharm. Des. 18:57355745 .

Goldammer, T., H. Zerbe, A. Molenaar, H. J. Schuberth, R. M. Brunner, S. R. Kata, and H. M. Seyfert. 2004. Mastitis increases mammary mRNA abundance of beta-defensin 5, toll-like-receptor 2 (TLR2), and TLR4 but not TLR9 in cattle. Clin. Diagn. Lab. Immunol. 11:174-185.

Halasa, T., K. Huijps, O. Osteras, and H. Hogeveen. 2007. Economic effects of bovine mastitis and mastitis management: A review. Vet. Q. 29:18-31.

Halliwell, B. 2007. Biochemistry of oxidative stress. Biochem. Soc. Trans. 35:1147-1150

Ingvartsen, K. L. 2006. Feeding- and management-related diseases in the transition cow: Physiological adaptations around calving and strategies to reduce feeding-related diseases. Anim. Feed Sci. Technol. 126:175-213.

Joumard-Cubizolles, L., J. C.-Y. Lee, C. Vigor, H. H. Leung, J. Bertrand-Michel, J.-M. Galano, A. Mazur, T. Durand, and C. Gladine. 2017. Insight into the contribution of isoprostanoids to the health effects of omega-3 PUFAs. Prostaglandins Other Lipid Mediat. 133:111-122.

Jungi, T. W., K. Farhat, I. A. Burgener, and D. Werling. 2011. Tolllike receptors in domestic animals. Cell Tissue Res. 343:107-120.

Klawitter, J., M. Haschke, T. Shokati, J. Klawitter, and U. Christians. 2011. Quantification of 15-F2t-isoprostane in human plasma and urine: Results from enzyme-linked immunoassay and liquid chromatography/tandem mass spectrometry cannot be compared. Rapid Commun. Mass Spectrom. 25:463-468.

Kuhn, H., S. Banthiya, and K. van Leyen. 2015. Mammalian lipoxygenases and their biological relevance. Biochim. Biophys. Acta 1851:308-330.

Kuhn, H., and V. B. O'Donnell. 2006. Inflammation and immune regulation by 12/15-lipoxygenases. Prog. Lipid Res. 45:334-356.

Kuhn, M. J., V. Mavangira, J. C. Gandy, C. Zhang, A. D. Jones, and L. M. Sordillo. 2017. Differences in the oxylipid profiles of bovine milk and plasma at different stages of lactation. J. Agric. Food Chem. 65:4980-4988.

Kumar, H., T. Kawai, and S. Akira. 2011. Pathogen recognition by the innate immune system. Int. Rev. Immunol. 30:16-34.
Lee, J. W., D. D. Bannerman, M. J. Paape, M. K. Huang, and X. Zhao. 2006. Characterization of cytokine expression in milk somatic cells during intramammary infections with Escherichia coli or Staphylococcus aureus by real-time PCR. Vet. Res. 37:219-229.

Lee, Y. Y., J. M. Galano, C. Oger, C. Vigor, R. Guillaume, J. Roy, J. Y. Le Guennec, T. Durand, and J. C. Lee. 2016. Assessment of isoprostanes in human plasma: Technical considerations and the use of mass spectrometry. Lipids 51:1217-1229.

Leslie, K. E., and C. S. Petersson-Wolfe. 2012. Assessment and management of pain in dairy cows with clinical mastitis. Vet. Clin. North Am. Food Anim. Pract. 28:289-305.

Liang, Y., Y. Zhou, and P. Shen. 2004. NF-kappaB and its regulation on the immune system. Cell. Mol. Immunol. 1:343-350.

Liu, Y., Y. Zhang, K. Schmelzer, T. S. Lee, X. Fang, Y. Zhu, A. A Spector, S. Gill, C. Morisseau, B. D. Hammock, and J. Y. Shyy. 2005. The antiinflammatory effect of laminar flow: The role of PPARgamma, epoxyeicosatrienoic acids, and soluble epoxide hydrolase. Proc. Natl. Acad. Sci. USA 102:16747-16752.

Maddox, J. F., C. C. Reddy, R. J. Eberhart, and R. W. Scholz. 1991. Dietary selenium effects on milk eicosanoid concentration in dairy cows during coliform mastitis. Prostaglandins 42:369-378.

Mattmiller, S. A., B. A. Carlson, J. C. Gandy, and L. M. Sordillo. 2014. Reduced macrophage selenoprotein expression alters oxidized lipid metabolite biosynthesis from arachidonic and linoleic acid. J. Nutr. Biochem. 25:647-654

Mattmiller, S. A., B. A. Carlson, and L. M. Sordillo. 2013. Regulation of inflammation by selenium and selenoproteins: Impact on eicosanoid biosynthesis. J. Nutr. Sci. 2:e28.

Mattos, R., C. R. Staples, A. Arteche, M. C. Wiltbank, F. J. Diaz T. C. Jenkins, and W. W. Thatcher. 2004. The effects of feeding fish oil on uterine secretion of PGF2alpha, milk composition, and metabolic status of periparturient Holstein cows. J. Dairy Sci. $87: 921-932$.

Mavangira, V., J. C. Gandy, C. Zhang, V. E. Ryman, A. Daniel Jones, and L. M. Sordillo. 2015. Polyunsaturated fatty acids influence differential biosynthesis of oxylipids and other lipid mediators during bovine coliform mastitis. J. Dairy Sci. 98:6202-6215.

Mavangira, V., M. J. Mangual, J. C. Gandy, and L. M. Sordillo. 2016. 15-F2t-Isoprostane concentrations and oxidant status in lactating dairy cattle with acute coliform mastitis. J. Vet. Intern. Med. $30: 339-347$

Mavangira, V., and L. M. Sordillo. 2018. Role of lipid mediators in the regulation of oxidative stress and inflammatory responses in dairy cattle. Res. Vet. Sci. https://doi.org/10.1016/j.rvsc. 2017.08.002.

Meirer, K., D. Steinhilber, and E. Proschak. 2014. Inhibitors of the arachidonic acid cascade: Interfering with multiple pathways. Basic Clin. Pharmacol. Toxicol. 114:83-91.

Menzies, M., and A. Ingham. 2006. Identification and expression of Toll-like receptors 1-10 in selected bovine and ovine tissues. Vet. Immunol. Immunopathol. 109:23-30.

Milne, G. L., Q. Dai, and L. J. Roberts 2nd. 2015. The isoprostanes-25 years later. Biochim. Biophys. Acta 1851:433-445.

Natarajan, R., and J. L. Nadler. 2004. Lipid inflammatory mediators in diabetic vascular disease. Arterioscler. Thromb. Vasc. Biol. 24:1542-1548

Pezeshki, A., P. Stordeur, H. Wallemacq, F. Schynts, M. Stevens, P. Boutet, L. J. Peelman, B. De Spiegeleer, L. Duchateau, F. Bureau, and C. Burvenich. 2011. Variation of inflammatory dynamics and mediators in primiparous cows after intramammary challenge with Escherichia coli. Vet. Res. 42:15.

Pinedo, P. J., A. De Vries, and D. W. Webb. 2010. Dynamics of culling risk with disposal codes reported by Dairy Herd Improvement dairy herds. J. Dairy Sci. 93:2250-2261.

Porcherie, A., P. Cunha, A. Trotereau, P. Roussel, F. B. Gilbert, P. Rainard, and P. Germon. 2012. Repertoire of Escherichia coli agonists sensed by innate immunity receptors of the bovine udder and mammary epithelial cells. Vet. Res. 43:14.

Pratico, D., J. A. Lawson, J. Rokach, and G. A. FitzGerald. 2001. The isoprostanes in biology and medicine. Trends Endocrinol. Metab. $12: 243-247$. 
Rao, P., and E. E. Knaus. 2008. Evolution of nonsteroidal anti-inflammatory drugs (NSAIDs): Cyclooxygenase (COX) inhibition and beyond. J. Pharm. Pharm. Sci. 11:81s-110s.

Raphael, W., L. Halbert, G. A. Contreras, and L. M. Sordillo. 2014. Association between polyunsaturated fatty acid-derived oxylipid biosynthesis and leukocyte inflammatory marker expression in periparturient dairy cows. J. Dairy Sci. 97:3615-3625.

Raphael, W., and L. M. Sordillo. 2013. Dietary polyunsaturated fatty acids and inflammation: The role of phospholipid biosynthesis. Int. J. Mol. Sci. 14:21167-21188.

Ricciotti, E., and G. A. FitzGerald. 2011. Prostaglandins and inflammation. Arterioscler. Thromb. Vasc. Biol. 31:986-1000.

Rose, D. M., S. N. Giri, J. S. Cullor, and R. B. Bushnell. 1991. The combined use of lipoxygenase and cyclooxygenase inhibitors in Klebsiella pneumoniae-induced bovine mastitis. Zentralbl. Veterinarmed. A 38:99-106.

Ryman, V. E., N. Packiriswamy, B. Norby, S. E. Schmidt, A. L. Lock, and L. M. Sordillo. 2017. Supplementation of linoleic acid (C18:2n -6) or alpha-linolenic acid (C18:3n-3) changes microbial agonistinduced oxylipid biosynthesis. J. Dairy Sci. 100:1870-1887.

Ryman, V. E., N. Packiriswamy, and L. M. Sordillo. 2015a. Role of endothelial cells in bovine mammary gland health and disease. Anim. Health Res. Rev. 16:135-149.

Ryman, V. E., N. Packiriswamy, and L. M. Sordillo. 2016. Apoptosis of endothelial cells by 13-HPODE contributes to impairment of endothelial barrier integrity. Mediators Inflamm. 2016:9867138

Ryman, V. E., G. M. Pighetti, J. D. Lippolis, J. C. Gandy, C. M. Applegate, and L. M. Sordillo. 2015b. Quantification of bovine oxylipids during intramammary Streptococcus uberis infection. Prostaglandins Other Lipid Mediat. 121(Pt B):207-217.

Serhan, C. N., and N. Chiang. 2008. Endogenous pro-resolving and anti-inflammatory lipid mediators: A new pharmacologic genus. Br. J. Pharmacol. 153(Suppl. 1):S200-S215.

Serhan, C. N., N. Chiang, and T. E. Van Dyke. 2008. Resolving inflammation: Dual anti-inflammatory and pro-resolution lipid mediators. Nat. Rev. Immunol. 8:349-361.

Soberman, R. J., T. W. Harper, D. Betteridge, R. A. Lewis, and K. F. Austen. 1985. Characterization and separation of the arachidonic acid 5-lipoxygenase and linoleic acid omega-6 lipoxygenase (arachidonic acid 15-lipoxygenase) of human polymorphonuclear leukocytes. J. Biol. Chem. 260:4508-4515.

Sordillo, L. M. 2013. Selenium-dependent regulation of oxidative stress and immunity in periparturient dairy cattle. Vet. Med. Int. 2013:154045

Sordillo, L. M. 2016. Nutritional strategies to optimize dairy cattle immunity. J. Dairy Sci. 99:4967-4982.

Sordillo, L. M., and S. L. Aitken. 2009. Impact of oxidative stress on the health and immune function of dairy cattle. Vet. Immunol. Immunopathol. 128:104-109.

Sordillo, L. M., and V. Mavangira. 2014. The nexus between nutrient metabolism, oxidative stress and inflammation in transition cows. Anim. Prod. Sci. 54:1204-1214.

Sordillo, L. M., N. O'Boyle, J. C. Gandy, C. M. Corl, and E. Hamilton. 2007. Shifts in thioredoxin reductase activity and oxidant status in mononuclear cells obtained from transition dairy cattle. J. Dairy Sci. 90:1186-1192

Sordillo, L. M., and J. E. Peel. 1992. Effect of interferon-gamma on the production of tumor necrosis factor during acute Escherichia coli mastitis. J. Dairy Sci. 75:2119-2125.
Sordillo, L. M., J. A. Weaver, Y. Z. Cao, C. Corl, M. J. Sylte, and I. K. Mullarky. 2005. Enhanced 15-HPETE production during oxidant stress induces apoptosis of endothelial cells. Prostaglandins Other Lipid Mediat. 76:19-34.

Spector, A. A., X. Fang, G. D. Snyder, and N. L. Weintraub. 2004. Epoxyeicosatrienoic acids (EETs): Metabolism and biochemical function. Prog. Lipid Res. 43:55-90.

Strandberg, Y., C. Gray, T. Vuocolo, L. Donaldson, M. Broadway, and R. Tellam. 2005. Lipopolysaccharide and lipoteichoic acid induce different innate immune responses in bovine mammary epithelial cells. Cytokine 31:72-86.

Tabas, I., and C. K. Glass. 2013. Anti-inflammatory therapy in chronic disease: Challenges and opportunities. Science 339:166-172.

Triggiani, M., F. Granata, A. Petraroli, S. Loffredo, A. Frattini, R. I. Staiano, G. Monaco, and G. Marone. 2009. Inhibition of secretory phospholipase $\mathrm{A}_{2}$-induced cytokine production in human lung macrophages by budesonide. Int. Arch. Allergy Immunol. 150:144-155

Trigona, W. L., I. K. Mullarky, Y. Cao, and L. M. Sordillo. 2006. Thioredoxin reductase regulates the induction of haem oxygenase-1 expression in aortic endothelial cells. Biochem. J. 394:207-216.

Vangroenweghe, F., L. Duchateau, P. Boutet, P. Lekeux, P. Rainard, M. J. Paape, and C. Burvenich. 2005. Effect of carprofen treatment following experimentally induced Escherichia coli mastitis in primiparous cows. J. Dairy Sci. 88:2361-2376.

Vernunft, A., T. Viergutz, C. Plinski, and J. M. Weitzel. 2014. Postpartum levels of 8-iso-prostaglandin F2alpha in plasma and milk phospholipid fractions as biomarker of oxidative stress in first-lactating dairy cows. Prostaglandins Other Lipid Mediat. 112:34-38.

Wada, M., C. J. DeLong, Y. H. Hong, C. J. Rieke, I. Song, R. S. Sidhu, C. Yuan, M. Warnock, A. H. Schmaier, C. Yokoyama, E. M. Smyth, S. J. Wilson, G. A. FitzGerald, R. M. Garavito, D. X. Sui, J. W. Regan, and W. L. Smith. 2007. Enzymes and receptors of prostaglandin pathways with arachidonic acid-derived versus eicosapentaenoic acid-derived substrates and products. J. Biol. Chem. 282:22254-22266.

Wang, Y., A. M. Armando, O. Quehenberger, C. Yan, and E. A. Dennis. 2014. Comprehensive ultra-performance liquid chromatographic separation and mass spectrometric analysis of eicosanoid metabolites in human samples. J. Chromatogr. A 1359:60-69.

Weaver, J. A., J. F. Maddox, Y. Z. Cao, I. K. Mullarky, and L. M. Sordillo. 2001. Increased 15-HPETE production decreases prostacyclin synthase activity during oxidant stress in aortic endothelial cells. Free Radic. Biol. Med. 30:299-308.

Wu, G.. J. M. Lu, W. A. van der Donk, R. J. Kulmacz, and A. L. Tsai. 2011. Cyclooxygenase reaction mechanism of prostaglandin $\mathrm{H}$ synthase from deuterium kinetic isotope effects. J. Inorg. Biochem. 105:382-390.

Yamada, T., M. Higuchi, and N. Nakanishi. 2013. Plasma 8-isoprostane concentrations and adipogenic and adipokine gene expression patterns in subcutaneous and mesenteric adipose tissues of fattening Wagyu cattle. J. Vet. Med. Sci. 75:1021-1027.

Yin, H., L. Xu, and N. A. Porter. 2011. Free radical lipid peroxidation: Mechanisms and analysis. Chem. Rev. 111:5944-5972.

Zia, S., S. N. Giri, J. Cullor, P. Emau, B. I. Osburn, and R. B. Bushnell. 1987. Role of eicosanoids, histamine, and serotonin in the pathogenesis of Klebsiella pneumoniae-induced bovine mastitis. Am. J. Vet. Res. 48:1617-1625. 